\title{
Planetary nebulae populations in external galaxies
}

\author{
Magda Arnaboldi $\mathbf{i}^{1,2}$ \\ ${ }^{1}$ European Southern Observatory, \\ Karl-Schwarzchild-Strasse 2, 85748 Garching bei München, Germany \\ email: marnabol@eso.org \\ ${ }^{2}$ INAF, Osservatorio Astronomico di Pino Torinese, \\ I-10025 Pino Torinese, Italy
}

\begin{abstract}
This review highlights the properties of the planetary nebulae in external galaxies as tracers of light, of the stellar population properties, and of the distances and kinematics of the parent galaxies. Recent results on the kinematics of the outer regions in giant elliptical galaxies and on the luminosity specific PN numbers (the $\alpha$ parameter) in these systems are presented, based on current surveys of planetary nebulae with the Planetary Nebulae Spectrograph (PN.S) and other instruments. Finally a brief discussion is given of planetary nebulae as tracers of the diffuse light in the nearby clusters, such as Virgo and Hydra I.
\end{abstract}

Keywords. planetary nebulae: general, galaxies: kinematics and dynamics

\section{Introduction}

Planetary Nebulae (PNs) are stars that evolve from the asymptotic giant branch (AGB) to their final destiny as white dwarfs. The majority of stars between 1 and $8 M_{\odot}$ evolve through a PN phase. As a consequence, in old stellar populations as those observed in bulges and early-type galaxies, most stars will reach the post AGB phase and a fraction of those stars will go through a PN phase, before ending their lives as white dwarfs.

In a $\mathrm{PN}$, the central star emits most of the light in the UV part of the spectrum. The nebular shell of the PN is able to convert the UV ionizing photons into various line emissions from the UV to the optical and down to the NIR. Up to $15 \%$ of the UV emitted energy by the central stars is re-emitted in the [OIII] $\lambda 5007 \AA$ line, the brightest optical emission of a PN (Dopita et al. 1992).

We can integrate the whole [OIII] flux emitted from a PN and derive the $m_{5007}$ magnitude defined as

$$
m_{5007}=-2.5 \log F_{5007}-13.74
$$

(Jacoby 1989). For a PN population observed in an external galaxy, we can derive the PN luminosity function (PNLF) for that population, which is empirically shown to follow an analytical formula

$$
N\left(m_{5007}\right)=C \times \mathrm{e}^{0.307 m_{5007}} \times\left[1-\mathrm{e}^{3\left(m^{*}-m_{5007}\right)}\right]
$$

(Ciardullo et al. 1998) where $m^{*}$ is the apparent magnitude of the bright cut-off. This analytic formula combines the observed behavior at the bright end, which is believed to originate from the most massive surviving stellar cores (Ciardullo et al. 1989), and the slow PN fading rate caused by the envelope expansion at the faint end (Henize \& Westerlund 1963). When this equation is integrated down $8 \mathrm{mag}$ from $\mathrm{m}^{*}$, it provides the total number $N_{\mathrm{PN}}$ of PNs associated with the total luminosity of a given galaxy. The 
magnitude range of $8 \mathrm{mag}$ from $m^{*}$ allows us to account for the faintest known Galactic planetary nebula (Ciardullo et al. 1989).

In external galaxies, $\mathrm{PN}$ are most often observed using a narrow band filter centered on the redshifted wavelength of the brightest emission line, the PN [OIII] $\lambda 5007 \AA$ line. In galaxies at distances larger than $2 \mathrm{Mpc}$, the $\mathrm{PN}$ emissions are spatially unresolved.

Planetary Nebulae trace light. The PN population in an external galaxy is expected to trace light because the luminosity-specific stellar death rate $(B)$ should be independent of the precise state of the underlying stellar population (Renzini \& Buzzoni 1986). Buzzoni et al. (2006) computed the specific evolutionary flux $B$ for simple stellar populations using different Initial Mass Functions (IMFs) and different ages, and measured a variation of $B$ by at most a factor of 2 . We can write the total number of PNs, $N_{\mathrm{PN}}$, associated with a given bolometric luminosity from a simple stellar population as

$$
N_{\mathrm{PN}}=B \times L_{\mathrm{TOT}} \times \tau_{\mathrm{PN}}
$$

where

- $N_{\mathrm{PN}}$ is the total number of PNs associated with the parent stellar population. $N_{\mathrm{PN}}$ is obtained by integration of the PNLF 8 mags down the bright cut off;

- $B$ is the luminosity-specific stellar death rate or specific evolutionary flux;

- $L_{\mathrm{TOT}}$ is the total bolometric luminosity of the parent simple stellar population;

- $\tau_{\mathrm{PN}}$ is the PN visibility lifetime, i.e. the time for the nebula to be detectable in either [OIII] or $\mathrm{H} \alpha$ surveys. The upper limit of $\tau_{\mathrm{PN}}$ is $30000 \mathrm{yr}$, that is the time required for a PN nebular envelope to fade entirely because of its expansion (Henize \& Westerlund 1963).

We can define the luminosity specific PN number, $\alpha$-parameter for short, as

$$
\alpha=\frac{N_{\mathrm{PN}}}{L_{\mathrm{TOT}}}=B \times \tau_{\mathrm{PN}}
$$

which provides us with a population averaged-measurement of the PN visibility lifetime $\tau_{\mathrm{PN}}$ for a $\mathrm{PN}$ population. Because of the small variations of the specific evolutionary flux $B$, variations of the observed $\alpha$ values indicate primarily different values of $\tau_{\mathrm{PN}}$ for PNs associated with the corresponding stellar populations.

Extended PN samples were collected for early-type galaxies (ETG) as part of the Planetary Nebulae Spectrograph (PN.S, Douglas et al. 2002) ETG survey

(http://www.astro.rug.nl/ pns/). In ETGs, the PN number density profile follows the surface brightness of the light distribution, as shown for the PN.S galaxies (Coccato et al. 2009) and for NGC 4697 (Méndez et al. 2001), M 60 (Teodorescu et al. 2011) and NGC 1399 (McNeil et al. 2010). Work is currently ongoing for a sample of S0s galaxies (Cortesi et al. 2011; A. Cortesi, 2012 Ph.D thesis).

Planetary Nebulae trace stellar populations. Buzzoni et al. (2006) investigated the time evolution of the luminosity specific PN number - the $\alpha$ parameter - using template galaxy models along the entire Hubble morphological sequence E-Sa-Sb-Sc-Sd-Im. The results indicate that the $\alpha$ parameter values predicted from these models depend on the mass loss relation during the evolution from the turn-off of the zero-age-main-sequence to the post-asymptotic giant branch phase; see Figure 7 in Buzzoni et al. (2006). When the $\alpha$ values are computed for the empirically calibrated Initial Final Mass Relation (IFMR) of Weidemann (2000), there results a flatter distribution from Im to E, see Figure 1.

Figure 1 compares the $\alpha$ model values with measurements from PN samples in ETGs and in galaxies from the Local Group. While model predictions and observed values are consistent for stellar populations in galaxy morphological types from Im to Sa, the observed $\alpha$ parameters in ETGs with red and old stellar populations are smaller than 


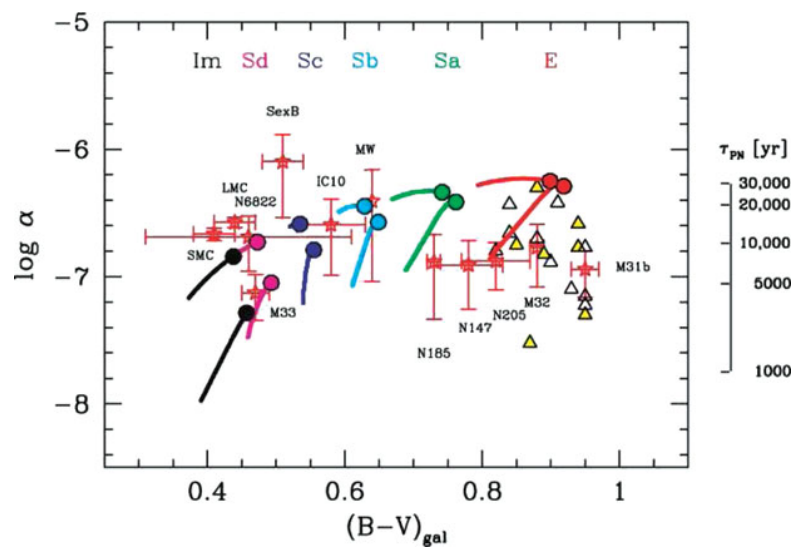

Figure 1. A comprehensive overview of the luminosity-specific PN number in Local Group galaxies (open star symbols) and external ellipticals (solid and open triangle symbols). Superposed on the plot, there are template galaxy models. Galaxy evolution along the whole E-Sa-Sb-Sc-Sd-Im Hubble morphological sequence is tracked by models from 5 to 15 Gyr, with the latter limit marked by the big solid dots. Two model sequences are reported on the plot assuming an IFMR as from the standard case of a la Reimers mass-loss parameter $\eta=0.3$ (lower sequence), and from the empirical relation of Weidemann (2000) (upper sequence). An indicative estimate of the mean representative PN lifetime (in years) is sketched on the right scale, according to Equation 1.4. From Buzzoni et al. (2006).

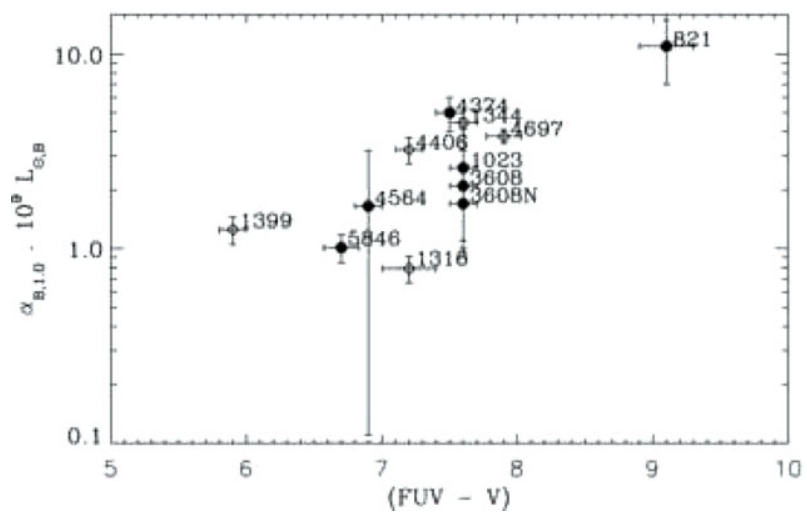

Figure 2. Correlation between the $\alpha_{B, 1.0}$ parameter and FUV-V color, measured from total extinction-corrected magnitudes FUV (from GALEX) and V (from RC3), from Coccato et al. (2009). Filled circles: sample galaxies for which $\alpha$ is calculated from the PN.S ETG sample data. Open diamonds: $\alpha$ values taken from Buzzoni et al. (2006).

the predicted values by up to a factor 7, see also Hui et al. (1993) and Ciardullo et al. (2005).

The relative PN deficiency in ETG supports the presence of a fraction of low-mass cores with $M_{\text {core }} \leqslant 0.55 \mathrm{M}_{\odot}$ : for such low mass cores, $\tau_{\mathrm{PN}}$ may become shorter because the time required for the excitation of the nebular envelope increases, and by the time it happens, the nebular shell may be near to being lost. Furthermore the part of the stellar population with $M_{\text {core }} \sim 0.52 \mathrm{M}_{\odot}$ may omit the PN phase entirely. These evolved stars may provide an enhanced contribution to the hotter horizontal branch (HB) and post-HB evolution as directly observed in M 32 and in the bulge of M 31, which in turn implies that the far UV flux in ETGs should anti-correlate with the measured $\alpha$ values. Such an anti-correlation is indeed observed in the PN.S ETG sample, see Figure 2. 
A possible external mechanism that can act on a $\mathrm{PN}$ to shorten its $\tau_{\mathrm{PN}}$ is the interaction with the hot intracluster medium. The ram pressure stripping by the hot high density gas in brightest cluster galaxies (BCGs) coupled with the large velocity of a PN through the medium can reduce the material in the PN envelope, therefore effectively reducing $\tau_{\mathrm{PN}}$ (Dopita et al. 2000, Villaver \& Stanghellini 2005).

Planetary Nebulae populations trace distances. The luminosity function of the [OIII] magnitudes associated with a PN population can be described by the simple analytical formula given in Eq. 1.2, whose absolute magnitude of the bright cut-off $M^{*}$ is observed to be invariant in different morphological types, from late to early types (Ciardullo et al. 2002). Because of the presence of a bright cut-off, the PNLF can be effectively used as a secondary distance indicator in both ETGs and late-type galaxies. One can ask whether the invariance of the PNLF bright cut-off for populations of different ages and metallicity can be explained on the basis of the evolution of simple stellar populations.

From single star evolution, we know that a PN's peak flux in the [OIII] $\lambda$ 5007 line is proportional to its core mass (Vassiliadis \& Wood 1994), the core mass is proportional to the turn-off mass (Kalirai et al. 2008), and the turn-off mass decreases with increasing age of the stellar population (Marigo et al. 2004). Therefore the absolute magnitude of the bright cut-off $M^{*}$ should become fainter in older and evolved stellar populations. The observed invariance of the PNLF bright cut-off indicates that the brightest PN may not be the end result of simple evolution of single stars, but rather have different progenitors, including blue stragglers (Ciardullo et al. 2005) and symbiotic stars (Soker 2006).

\section{Planetary nebulae as kinematical tracers}

In addition to measuring the $m_{5007}$ magnitude and determining the distance to a given galaxy via the PNLF, the [OIII] $\lambda 5007 \AA$ emission allows us to measure of the line-ofsight (LOS) velocity of a PN. By measuring $v_{\mathrm{LOS}}$ for the PN population in the outer regions of galaxies we can obtain the kinematics of the stellar population at large radii and derive the projected two-dimensional velocity field.

Since the work of Arnaboldi et al. (1994) on NGC 1399 and of Hui et al. (1995) on Centaurus A (NGC 5128), there has been a considerable success in mapping the kinematics of the stellar population in the outer regions of ETGs out to $5 R_{e}$ (Arnaboldi et al. 1996; Arnaboldi et al. 1998; Méndez et al. 2001; Romanowsky et al. 2003; Coccato et al. 2009; Napolitano et al. 2009; McNeil et al. 2010; Napolitano et al. 2011; Teodorescu et al. 2011; Cortesi et al. 2011), and intracluster light (ICL) in nearby clusters (Arnaboldi et al. 2004; Gerhard et al. 2005; Gerhard et al. 2007; Doherty et al. 2009; Ventimiglia et al. 2011).

In the galaxies studied thus far, the PN density and kinematics is everywhere consistent with the integrated light measurements within the errors (Coccato et al. 2009), with the possible exception of NGC 4697 (Sambhus et al. 2006). The outer halos kinematics from the PN samples in ETGs show the following properties:

- there is a dichotomy in the outer halo kinematics, the velocity dispersion profile is either slowly falling or rapidly falling (Coccato et al. 2009) with increasing radius.

- The rotational properties of the halos traced by the ratio $v / \sigma$ where $v$ is the circular velocity and $\sigma$ is the velocity dispersion correlates with that within $R_{e}$ for the greater part of the sample galaxies observed so far.

- The slow/fast rotators division in the outer halos (Coccato et al. 2009) is similar as in the cores (Emsellem et al. 2007). There are some more complicated cases, as kinematic misalignment is more frequent at large radii (Shih \& Méndez 2010, Teodorescu et al. 2011). 
- A fraction of morphologically-undisturbed ellipticals show large distortions of their kinematics or presence of kinematic sub-components at large radii, as in the outer halos of NGC 1399 (McNeil et al. 2010) and NGC 3311 (Ventimiglia et al. 2011).

\section{The brightest planetary nebulae in the Virgo Cluster}

The PN populations in the core of the Virgo cluster are studied via narrow band imaging surveys, using the "on-band, off-band" technique, in which a field is imaged through a narrow band filter, centered at the redshifted [OIII] $\lambda 5007 \AA$ emission in these clusters, and a broad band filter. PN candidates are those unresolved sources that have a color excess in [OIII] and no detected continuum (Arnaboldi et al. 2002). Tens of intracluster PN (IPN) were discovered in the Virgo cluster, in its core and in outer targeted fields (Arnaboldi et al. 2002; Okamura et al. 2002; Arnaboldi et al. 2003; Feldmeier et al. 2003; Feldmeier et al. 2004; Aguerri et al. 2005; Castro-Rodriguez et al. 2009). Such surveys are complete $0.5 \mathrm{mag}$ down the PNLF, therefore they sample mostly the brightest PNs in these stellar populations. The spectroscopic follow-up with multi-object (Arnaboldi et al. 2003) and multi fiber spectrographs (Arnaboldi et al. 2004; Doherty et al. 2009) allow

- to detect the second [OIII] $\lambda 4959 \AA$ emission besides $\lambda 5007 \AA$ in single spectra,

- measure the radial velocities and associate PNs with the dynamical components along the LOS,

- determine PN physical parameters, the $m_{5007}$ and the velocity $v_{\mathrm{EXP}}$ derived from the FWHM of the [OIII] $\lambda 5007 \AA$ emission, i.e. the expansion velocity of a PN nebular envelope. These PN physical parameters can be correlated with those of its parent stellar population (Arnaboldi et al. 2008).

In case of M87, the BCG in the Virgo cluster, the PN LOS velocity distribution traces the M87 halo out to $150 \mathrm{kpc}$ and shows that the halo PN population has a slightly brighter cut-off than that in the galaxy's central region, by $0.3-0.4 \mathrm{mag}$, see the observed M87 PNLF in Figure 3. Because the LOS distance to these PNs is known, via the physical association with the M87 halo, their absolute magnitude is also known, and so are then the core masses required to power the detected [OIII] $\lambda 5007 \AA$ fluxes: for the brightest $\mathrm{PN}$ in the M87 halo, the core masses are $\simeq 0.62 \mathrm{M}_{\odot}$. The IFMR for solar metallicity stars predicts that $\mathrm{PN}$ progenitors with $2.2 \mathrm{M}_{\odot}$ give final core masses of $0.62 \mathrm{M}_{\odot}$. Turnoff masses of $\simeq 2 \mathrm{M}_{\odot}$ belong to populations with ages $\sim 1$ Gyr. It is unlikely that such progenitors exist in the Virgo core, as the stellar population is mostly old (Williams et al. 2007): therefore close binaries and blue stragglers are indicated as most likely progenitors for these intrinsically bright PNs.

\section{The brightest planetary nebulae in the Hydra I Cluster}

The multi-slit imaging spectroscopy technique. The nearest clusters beyond Virgo and Fornax are at distances of about $50 \mathrm{Mpc}$, and the brightest PN in the Hydra I and the Centaurus clusters have fluxes of $8 \times 10^{-18} \mathrm{erg} \mathrm{s}^{-1} \mathrm{~cm}^{-2}$. They cannot be detected using standard narrow band imaging because the noise in the $40-60 \AA$ sky is of the same order of the signal we want to detect.

A successful technique to detect the [OIII] $\lambda 5007 \AA$ emission from the brightest PNs in these clusters is the "Multi-Slit Imaging Spectroscopy technique" (MSIS, Gerhard et al. 2005; Arnaboldi et al. 2007). This is a blind search technique that combines a mask of parallel multiple slits with a narrow band filter, centered at the redshifted [OIII] emission of the PNs in these clusters, plus a dispersing element to obtain spectra of all PNs that 


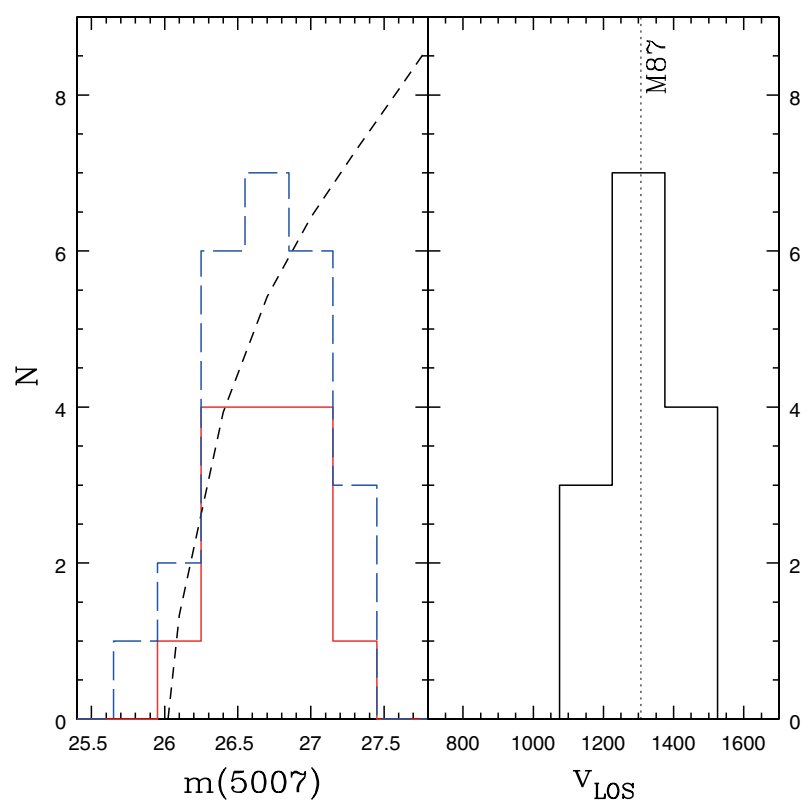

Figure 3. Left panel: the observed PNLF in the Virgo cluster core fields, in 0.3 mag bins. Long dashed blue line: PNLF for the entire spectroscopically confirmed sample of PNs in the Virgo cluster core. Red continuous line: PNLF for the M87 halo PNs. Short dashed black line: analytical formula for the PNLF with $m^{*}=26.0$. Right panel: LOS velocity distribution for the 14 M87 halo PNs. The systemic velocity of M87 is also indicated. From Arnaboldi et al. (2008).

lie behind the slits. The sky noise at the emission line of a PN comes from a few $\AA$ only, depending on slit width and spectral resolution (Gerhard et al. 2005).

$P N$ population in the halo of NGC 3311. The MSIS observations of the Hydra I cluster carried out with the FORS2 spectrograph at the VLT detected 56 PNs and 26 background galaxies in a $6^{\prime} .8 \times 6^{\prime} .8$ field centered on the BCG NGC 3311 in the cluster core (Ventimiglia et al. 2011). In addition to the study of the halo kinematics, Ventimiglia et al. (2011) measured the luminosity specific PN number $\alpha$ for the stellar population in the extended NGC 3311 halo, and obtain a value that is a factor 4-6 lower than the predicted value of $\log \alpha=-7.30$ from the $\alpha$ vs. FUV-V color correlation for the ETG populations shown in Figure 2. In Figure 4 the observed cumulative PN number for the NGC 3311 halo (green line) and the total NGC 3311 plus ICL light (black line) in the Hydra I core are plotted together with the predicted cumulative PN number for the NGC 3311 plus ICL (using $\log \alpha=-7.30$, red line), as function of the radial distance from the center of NGC 3311. The large discrepancy between the red and green lines indicates a decrement of PNs in the NGC 3311 halo. This PN deficit may signal that additional effects are at work that shape the properties of the PN population in the NGC 3311 halo.

A possibility is that ram pressure against the hot X-ray emitting gas in the halo of NGC 3311 is high enough to severely shorten $\tau_{P N}$, the visibility time of a PN, see Introduction. With a density of the intracluster medium (ICM) inside $5^{\prime}$ around NGC 3311 of about $\rho_{\mathrm{ICM}} \simeq 6 \times 10^{-3} \mathrm{~cm}^{-3}$ and a typical velocity of $v=\sqrt{3} \times 450 \mathrm{~km} \mathrm{~s}^{-1} \simeq$ $800 \mathrm{~km} \mathrm{~s}^{-1}$, the ram pressure on the NGC 3311 halo PNs is $\sim 40$ times stronger than in the simulated case by Villaver \& Stanghellini (2005) for the Virgo cluster. The ram pressure effect could be much stronger in the Hydra I cluster and shorten significantly $\tau_{\mathrm{PN}}$ for the NGC 3311 halo population. 


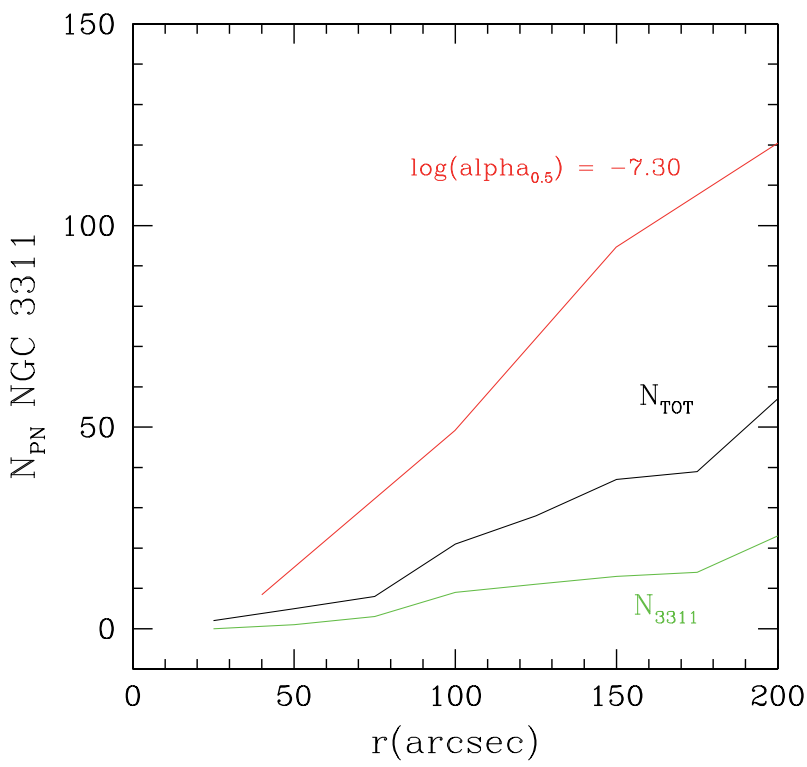

Figure 4. Observed and predicted cumulative PN number for the NGC 3311 halo and the total (NGC 3311 plus ICL) light in the Hydra I core, as function of the radial distance from the center of NGC 3311. Red line: predicted cumulative number of PNs computed using $\log \alpha=-7.30$. Green line: measured cumulative number of PNs associated with the NGC 3311 halo on the basis of the $v_{\text {LOS }}$. Black line: measured cumulative number of PNs corresponding to the total light in the Hydra I core. From Ventimiglia et al. (2011).

\section{Summary and conclusions}

In this review we illustrate that PN populations are ubiquitous to the light associated with external galaxies and the diffuse light in the cores of nearby clusters. Deep photometry and kinematics of PN populations show that they trace star light and motions in the extended, luminous halos of BCGs and ICL.

The PN population shows dependencies on the stellar population age and metallicity. Recent observations of PNs in the Hydra I cluster indicate that there may be an additional effect by the hot ICM, which could decrease the visibility lifetime $\tau_{\mathrm{PN}}$ of PNs. Further theoretical and observational work is needed to quantify effects of stellar population age, metallicity and ICM on both the PNLF bright cut-off and the luminosity specific PN number $\alpha$.

The study of the PNs in the ICL and the halos of galaxies is important for constraining how and when these components formed. In the case of the Hydra I cluster the results indicate that the build-up of the BCG halo and the nearby ICL continues, and that the formation of the outer halo and ICL is an on-going and long lasting process.

\section{Acknowledgements}

I wish to thank my collaborators for their enthusiasm and support and the organizers for inviting me to give a review on $\mathrm{PN}$ populations in external galaxies at the IAU Symposium 283.

\section{References}

Aguerri, J. A. L., Gerhard, O. E., Arnaboldi, M., Napolitano, N. R., et al. 2005, AJ, 129, 2585 Arnaboldi, M., Freeman, K. C., Hui, X., Capaccioli, M., et al. 1994, Messenger, 76, 40 
Arnaboldi, M., Freeman, K. C., Méndez, R. H., Capaccioli, M., et al. 1996, ApJ, 472, 145

Arnaboldi, M., Freeman, K. C., Gerhard, O. E., Matthias, M., et al. 1998, ApJ, 507, 759

Arnaboldi, M., Aguerri, J. A. L., Napolitano, N. R., Gerhard, O. E., et al. 2002, AJ, 123, 760

Arnaboldi, M., Freeman, K. C., Okamura, S., Yasuda, N., et al. 2003, AJ, 125, 514

Arnaboldi, M., Gerhard, O. E., Aguerri, J. A. L., Freeman, K. C., et al. 2004, ApJ, 614, L33

Arnaboldi, M., Gerhard, O. E., Okamura, S., Kashikawa, N. et al. 2007, PASJ, 59, 419

Arnaboldi, M., Doherty, M., Gerhard, O. E., Ciardullo, R., et al. 2008, ApJ, 674, L17

Buzzoni, A., Arnaboldi, M., \& Corradi, R. L. M. 2006, MNRAS, 368, 877

Castro-Rodriguez, N., Arnaboldi, M., Aguerri, J. A. L., Gerhard, O. E., et al. 2009, A\&A, 507, 621

Ciardullo, R., Jacoby, G. H., Ford, H. C., \& Neill, J. D. 1989, ApJ, 339, 53

Ciardullo, R., Jacoby, G. H., Feldmeier, J. J., \& Bartlett, R. E. 1998, ApJ, 492, 62

Ciardullo, R., Feldmeier, J. J., Jacoby, G. H., Kuzio de Naray, R., et al. 2002, ApJ, 577, 31

Ciardullo, R., Sigurdsson, S., Feldmeier, J. J., \& Jacoby, G. H. 2005, ApJ, 629, 499

Coccato, L., Gerhard, O. E., Arnaboldi, M., Das, P., et al., 2009, MNRAS, 394, 1249

Cortesi, A., Merrifield, M. R., Arnaboldi, M., Gerhard, O. E., et al. 2011, MNRAS, 414, 642

Doherty, M., Arnaboldi, M., Das, P., Gerhard, O. E., et al. 2009, A\& $A$, 502, 771

Dopita, M., Massaglia, S., Bodo, G., Arnaboldi, M. et al. 2000, ASPC, 199, 423

Dopita, M., Jacoby, G. H., \& Vassiliadis, E. 1992, ApJ, 389, 27

Douglas, N. G., Arnaboldi, M., Freeman, K. C., Kuijken, K., et al. 2002, PASP, 114, 1234

Emsellem, E. Cappellari, M., Krajnović, D., van de Ven, G., et al. 2007, MNRAS, 379, 401

Feldmeier, J. J., Ciardullo, R., Jacoby, G. H., \& Durrell, P. R. 2003, ApJS, 145, 65

Feldmeier, J. J., Ciardullo, R., Jacoby, G. H., \& Durrell, P. R. 2004, ApJ, 615, 196

Gerhard, O. E., Arnaboldi, M., Freeman, K. C., Kashikawa, N., et al. 2005, ApJ, 621, L93

Gerhard, O. E., Arnaboldi, M., Freeman, K. C., Okamura, S., et al. 2007, A\&\&A, 498, 815

Jacoby, G. H. 1989, ApJ, 339, 39

Hui, X., Ford, H. C., Ciardullo, R., \& Jacoby, G. H. 1993, ApJ, 414, 463

Hui, X., Ford, H. C., Freeman, K. C., \& Dopita, M. 1995, ApJ, 449, 592

Henize, K. G. \& Westerlund, B. E. 1963, ApJ, 137, 747

Kalirai, J. S., Hansen, B. M. S., Kelson, D. D., Reitzel, D. B., et al. 2008, ApJ, 676, 594

Marigo, P., Girardi, L., Weiss, A., Groenewegen, M. A. T., et al. 2004, A\& A, 423, 995

McNeil, E., Arnaboldi, M., Freeman, K. C., Gerhard, O. E., et al. 2010, A\&J A, 518, 44

Méndez, R. H., Riffeser, A., Kudritzki, R. P., Matthias, M. et al. 2001, ApJ, 563, 135

Napolitano, N. R., Romanowsky, A. J., Coccato, L., Capaccioli, M., et al. 2009, MNRAS, 393, 329

Napolitano, N. R., Romanowsky, A. J., Capaccioli, M., Douglas, N. G., et al. 2011, MNRAS, 411, 2035

Okamura, S., Yasuda, N., Arnaboldi, M., Freeman, K. C., et al. 2002, PASJ, 54, 883

Renzini, A. \& Buzzoni, A. 1986, ASSL, 122, 195

Romanowsky, A. J., Douglas, N. G., Arnaboldi, M., Kuijken, K., et al. 2003, Science, 301, 1696

Sambhus, N., Gerhard, O. E., \& Méndez, R. H. 2006, AJ, 131, 837

Shih, H. Y. \& Méndez, R. H. 2010, ApJ, 725, L97

Soker, N. 2006, ApJ, 645, L57

Teodorescu, A. M., Méndez R. H., Bernardi, F., Thomas, J., et al. 2011, ApJ, 736, 65

Vassiliadis, E. \& Wood, P. 1994, ApJS, 92, 125

Ventimiglia, G., Arnaboldi, M., \& Gerhard, O. E. 2011 A $\mathcal{E}$ A, 528, 24

Villaver, E. \& Stanghellini, L. 2005 ApJ, 632, 854

Weidemann, V. 2000, A\&\&A, 363, 647

Williams, B. F., Ciardullo, R., Durrell, P. R., Vinciguerra, M., et al. 2007, ApJ, 656, 756 